\title{
Societal bias reinforcement through machine learning: a credit scoring perspective
}

\author{
Bertrand K. Hassani ${ }^{1,2}$
}

Received: 3 July 2020 / Accepted: 30 October 2020 / Published online: 18 December 2020

(c) The Author(s) 2020

\begin{abstract}
Does machine learning and AI ensure that social biases thrive? This paper aims to analyze this issue. Indeed, as algorithms are informed by data, if these are corrupted, from a social bias perspective, good machine learning algorithms would learn from the data provided and reverberate the patterns learnt on the predictions related to either the classification or the regression intended. In other words, the way society behaves whether positively or negatively would necessarily be reflected by the models. In this paper, we analyze how social biases are transmitted from the data into banks loan approvals by predicting either the gender or the ethnicity of the customers using the exact same information provided by customers' through their applications
\end{abstract}

Keywords SMOTE $\cdot$ Machine learning $\cdot$ Social bias $\cdot$ Credit scoring $\cdot$ Random forest

\section{Introduction}

According to the Cambridge dictionary, ${ }^{1}$ a bias implies the "action of supporting or opposing a particular person or thing in an unfair way". These biases might be unconscious, i.e., the person with the bias is not aware of it, or worst, this bias might just be the result of conforming to the norm, as norms are behaviors that are self-enforcing at the group level and are not necessarily positive as it is just something followed by the masses. Social biases, to be precise, occur when we unknowingly or deliberately make a judgment about certain individuals, groups, races, opinion, and so on, due to preconceived notions about the group. These can either be positive or negative beliefs and are often instilled in us based on our own culture and environment. Societal biases, in turn, occur when social biases become the norm.

Social biases have been reported in many papers either released by NGOs; for instance see $[5,6,8]$, or academics see [7] or [4] among others. ${ }^{2}$ As reported in the aforementioned papers, it is clear that both gender gaps and ethnicity

Bertrand K. Hassani

bertrand.hassani@gmail.com

1 Université Paris 1 Panthéon-Sorbonne, CES106 bd de l'Hôpital, 75013 Paris, France

2 University College London - Computer Science, 66-72 Gower Street, London WC1E 6EA, UK gaps exist in remuneration; thus, it would not be surprising that these gaps have impacts on consumption, education, or access to loans, though this remains to be proved. This is the objective of this paper using data sets, capturing both gender and ethnicity (among other elements), traditionally used for scoring purposes.

As algorithms learn from data, if these are corrupted, from a social bias perspective, not necessarily from a data quality point of view, then a good machine learning algorithm would learn from the data provided and reverberate the patterns learnt onto the predictions related either to the classifications or the regressions intended. Therefore, if the data sets are capturing the way society behaves whether it is positive or negative (discrimination towards gender, ethnicity, among others), then this would be reflected by the models; for instance, if someone faces discrimination in their workplace, then this is likely to be reverberated in her remuneration and mechanically in her access to loans; and a "good" algorithm will naturally score these discriminated people at a lower level.

Essentially, machine learning captures all features characterizing a phenomenon and then relies on them to make predictions. However, these features may characterize not only the intended phenomenon, but might also be informative

\footnotetext{
1 https://dictionary.cambridge.org/dictionary/english/bias.

2 Numerous sources from both Public Policy and Law departments of Universities, in particular, have been identified.
} 
Fig. 1 This table provides the descriptive statistics of the "Ethnicity Set". The data set contains information about the income of the applicants, current rating, credit limit, the number of credit cards they possess, age, level of education, gender, marital status, ethnicity, and current balance. This data set contains 400 data points. Among these 400 data points, 99 are classified as "AfricanAmerican", 102 are classified as "Asian", and 199 are as "Caucasian". The sample age ranged from 23 to 98 are fairly split. Roughly half the sample represents women (207) and the other half men (193)

\begin{tabular}{|c|c|c|c|}
\hline \multicolumn{2}{|c|}{ Income } & \multicolumn{2}{|c|}{ Limit } \\
\hline Min. & 12 & Min. : & 855 \\
\hline Lst Qu. & 16481 & 1st Qu.: & 3088 \\
\hline Median & 29715 & Median : & 4622 \\
\hline Mean & 4067 & Mean : & 4736 \\
\hline 3rd Qu. & 53778 & 3rd Qu. : & 5873 \\
\hline $\operatorname{Max}$. & 186634 & $\operatorname{Max} . \quad:$ & 13913 \\
\hline Married & & Ethn & icity \\
\hline No : 155 & Afric & America & $n: 99$ \\
\hline Yes: 245 & Asian & & $: 102$ \\
\hline & cauc & & :199 \\
\hline
\end{tabular}

Rating

Min. : 93.0

1st Qu.:247.2

Median : 344.0

Mean : 354.9

3rd Qu. : 437.2

Max. : 982.0 Balance

Min. : 0.00

1st Qu.: 68.75

Median : 459.50

Mean : 520.01

3rd Qu.: 863.00

Max. : :1999.00

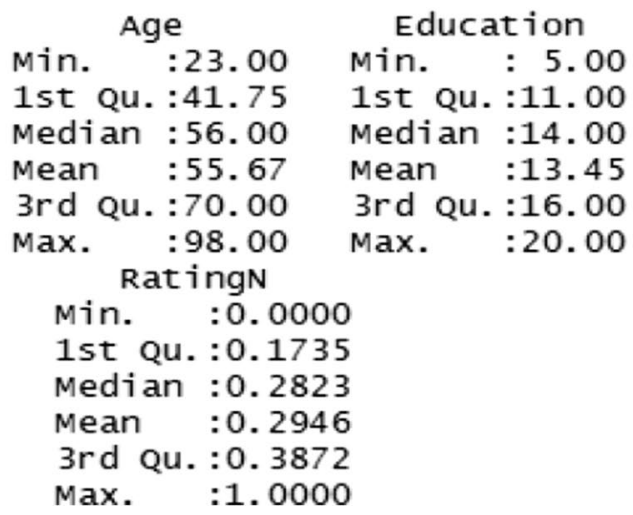

Cards
Min. $: 1.000$
1st Qu. $: 2.000$
Median $: 3.000$
Mean $: 2.958$
3rd Qu. $: 4.000$
Max. $: 9.000$
Ethnic
Caucasian:199
Other $: 201$

student

No : 360

Yes: 40 in characterizing other phenomena, categories of features, or classes. For instance, someone with a low income may be related to the fact that her loan request is not approved, but may also reflect that they are relatively poor, having a "blue collar" job as well as their gender, ethnicity, location of their home, and so on. In other words, social biases are naturally and mechanically captured in data, and therefore, we believe that these might be captured and replicated by machine learning algorithms. If these algorithms are used for loan approval or credit scoring purposes, then they might not only replicate these biases, but may also validate them as normal and transmit them over time. Indeed, the newest data would be subsequently used to assess future customers' requests. Furthermore, financial institutions profit generating paradigm and regulations have the negative effect of ensuring that the system cannot self correct. Certainly, prudential rules do not allow financial institutions to take more risk than what is prescribed [9].

Therefore, in this paper, we intend to address the following. Assuming that a credit scoring model is not socially biased, the data used to assess the suitability of a loan applicant should not give any information regarding either their gender or their ethnicity. However, we have seen from numerous reports that the world thrives with inequality, for instance, inequality in remuneration. It is generally accepted that income is one of the main elements for a bank to accept a loan request. Therefore, in this paper, using credit data, we will try to predict both the ethnicity of the applicants and their gender. We assume that if we are able to predict either of these from a data set used for credit scoring, then it means that the intrinsic characteristics of each population will be spilled over on their access to loans. Thus, it would be necessary to correct the rating for the bias identified while ensuring that regulatory requirements are fulfilled.

In this paper, after presenting the data sets, we will introduce the methodology and discuss the results obtained. A last section offers a conclusion.

\section{The data sets}

Two data sets used for scoring purposes provided by financial institutions are used in what follows. These data sets contain information about both gender and ethnicity. These data sets are publicly available on either the Kaggle website or UCL's Github. Figures 1 and 4 provide numerous statistical pieces of information regarding the fields of each data set, such as distributions, number of elements per category, and so on. It is important to mention that though one of the data sets contains both ethnicity and gender, we opted for two different data sets to ensure the robustness of our analysis by not relying on a single set of information. 
Table 1 Quartiles of African-American, Asian, and Caucasian distributions of income

\begin{tabular}{|c|c|c|c|c|}
\hline $0 \%$ & $25 \%$ & $50 \%$ & $75 \%$ & $100 \%$ \\
\hline \multicolumn{5}{|c|}{ African-American } \\
\hline 1409 & 19,445 & 33,017 & 54,860 & 186,634 \\
\hline \multicolumn{5}{|l|}{ Asian } \\
\hline 177 & 15,514 & 27,732 & 52,958 & 180,379 \\
\hline \multicolumn{5}{|c|}{ Caucasian } \\
\hline 12 & 16,293 & 30,002 & 53,943 & 182,728 \\
\hline
\end{tabular}

\subsection{The ethnicity set}

The first data set, referred to as the "Ethnicity Set", ${ }^{3}$ contains information about the income of the applicants, current rating, credit limit, the number of credit cards they possess, age, level of education, gender, marital status, ethnicity, and current balance. This data set contains 400 data points. Among these 400 data points, 99 are classified as "African-American", 102 are classified as "Asian", and 199 are classified as "Caucasian". The sample age ranged from 23 to 98 are fairly split. Roughly half the sample represents women (207) and the other half men (193). Figure 1 provides detailed information pertaining to each field included in the data set.

In the considered sample, the average income for African-Americans is 44,698.37, Asians is 40,144.45, and Caucasians $38,939.95$ dollars. The quartiles representing the income distribution of each ethnic group are represented in Table 1.

We see in the ethnicity data that the three groups are having fairly similar distributions of income. As such, it is not representative of what has been reported in the various reports aforementioned. Therefore, after working on the data set as obtained, we will also analyze the impact of modifying the income of these groups changing the income by a certain coefficient to better reflect what has been reported by NGOs, as such we will create an alternate data set where AfricanAmericans are earning 25\% less than Caucasians and Asians are earning $10 \%$ less than Caucasians bringing the average down to 33,523.78 for African-Americans and 36,130.01 for Asians. In Table 2, the quartiles of the modified data are provided. Figures 2 and 3 depict the histogram, respectively, related to the original "Ethnicity Set" and the modified one.

\footnotetext{
3 The data are available at https://www.kaggle.com/suzanaiacob/ predicting-credit-card-balance-using-regression.
}

Table 2 Quartiles of African-American, Asian, and Caucasian distributions of income, after alteration of the data set

\begin{tabular}{|c|c|c|c|c|}
\hline $0 \%$ & $25 \%$ & $50 \%$ & $75 \%$ & $100 \%$ \\
\hline \multicolumn{5}{|c|}{ African-American } \\
\hline 1057 & 14,583 & 24,763 & 41,145 & 139,976 \\
\hline \multicolumn{5}{|l|}{ Asian } \\
\hline 159 & 13,963 & 24,959 & 47,662 & 162,341 \\
\hline \multicolumn{5}{|c|}{ Caucasian } \\
\hline 12 & 16,293 & 30,002 & 53,943 & 182,728 \\
\hline
\end{tabular}

\subsection{The gender set}

The second data set, referred to as the "Gender Set", ${ }^{4}$ contains information about the gender of the applicants, marital status, whether they have dependents, level of education, whether they are self employed or not, income, income of the co-applicant, the amount of the loan requested, the term of the requested loan, credit history, the location of their current property, and the status of their loan. This data set contains 597 data points; 113 of these represent women and 484 of these represent man. $31 \%$ of the applications contained in the data set have led to a refusal (Fig. 4).

In Fig. 5, the income by gender has been represented; as can be observed, the sample is consistent with what has been reported internationally; there is a clear gap in terms of remuneration, and women are clearly earning less than men on average. Unfortunately, since the type of employment is not shown, it is not possible to investigate the matter further, but there is no reason why this should affect our reasoning, as any inequality would be reflected accordingly. Indeed, the average monthly income of women in the data set considered, we observed an average of 4530.468 dollars, while, for men, this average went up to 5769.968 dollars, i.e., a difference of $27.36 \%$. The quantiles representing the income distribution are provided in Table 3.

\section{Methodology}

In this paper, we assume that for a credit scoring data set to be unbiased, the information provided should not contain any direct or indirect information susceptible to give away the gender or the ethnic group of customers. Therefore, our main objective is to try to figure out or predict either the gender or the ethnicity of customers based on data used for credit scoring purposes. Though this paper would gain

\footnotetext{
4 The data are available at https://www.kaggle.com/ajaymanwani/ loan-approval-prediction, https://github.com/shrikant-temburwar/ Loan-Prediction-Dataset.
} 
Combined

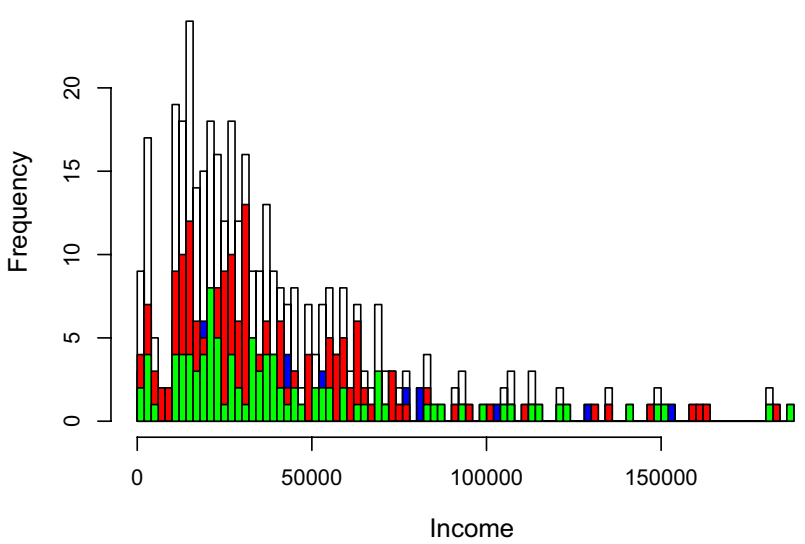

Caucasian

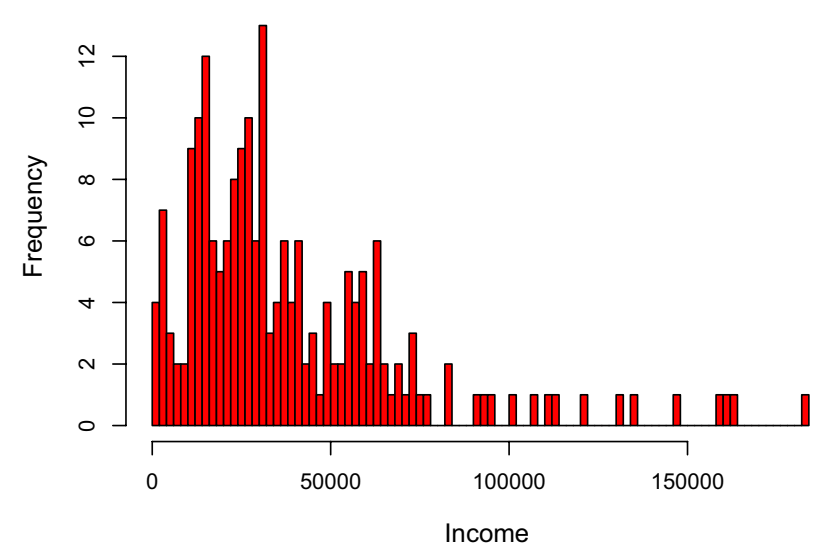

Fig. 2 This figure presents four histograms. On the top left-end corner, the various groups are represented simultaneously. The distribution of income of the whole data set is depicted along the distribution

from being tested on larger or different data sets, the results obtained implementing the following approaches are easily extendable. Furthermore, it is worth mentioning that though, in most countries, the ethnicity of customers is not given, if the data contain information characteristic of a certain group (for instance the level of remuneration), then not having an explicit field does not solve the problem. However, the fact that a field explicitly either states the gender or the ethnicity of the customer permits testing our hypothesis. In what follows, we will proceed in three steps:

1. The first step is to test whether the data are actually usable for credit scoring purposes. In other words, we are going to test if it is possible to perform a regression to predict the scores using the "Ethnicity Set" and if it is possible to perform a classification to predict whether their application will be approved or not using the "Gender Set".
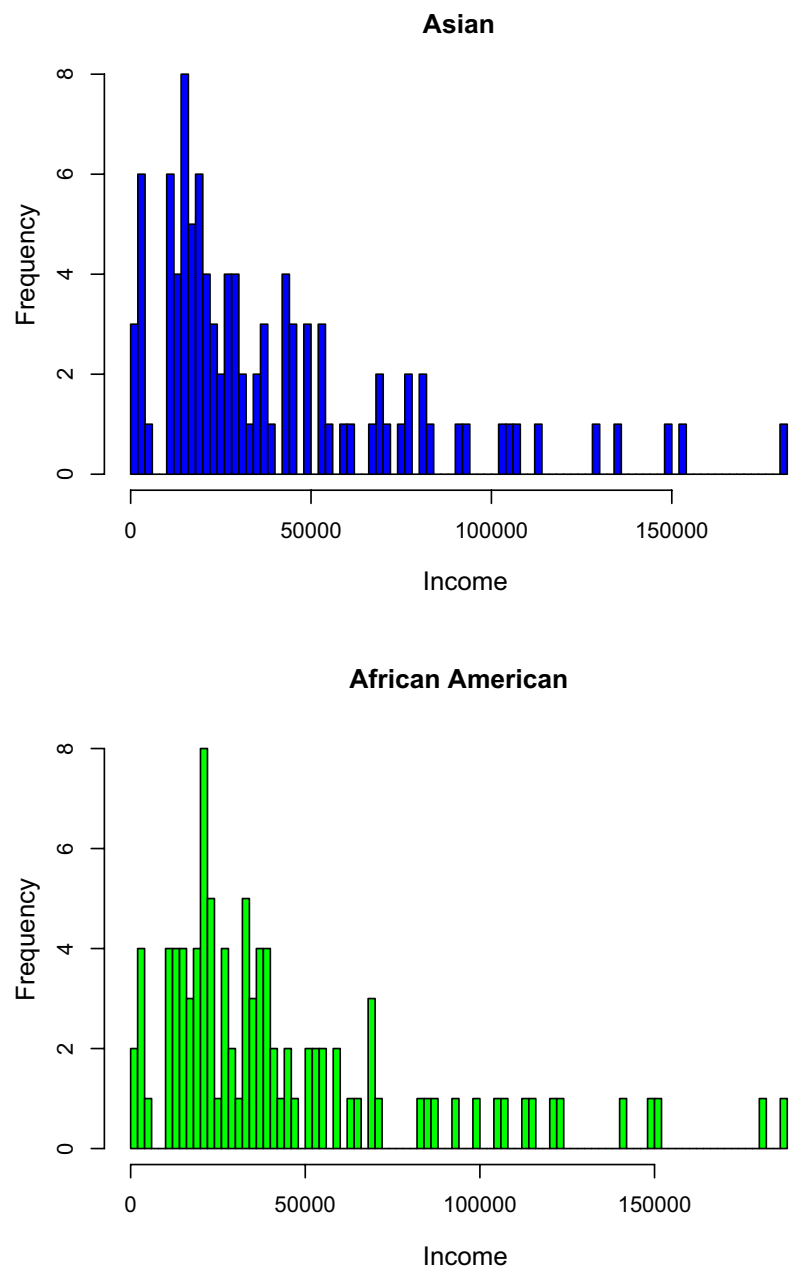

of income of each ethnic group. The three other histograms depict the distribution of income for each ethnic group, i.e., Caucasian, AfricanAmerican, and Asian

2. In a second step, we will try to predict either the gender or the ethnicity of the customers contained in the database.

3. In a third step, we will try to improve the prediction.

When the variable to be predicted is continuous, we will perform a regression. When the response variable is discreet, we will perform a classification. A similar algorithm can be used in both situations. Following [1, 3], we initially used a random forest growing 750 trees. Random forests operate by constructing a multitude of decision trees at training time and producing the class as the output according to the mode of the classes or the mean prediction of each individual tree, respectively. Random forests correct for decision trees overfitting tendency [2].

To evaluate the quality of the regression, we will use the mean-squared error (mse) and for the classifications the F1-Score which is equal to $2 \times \frac{\text { precision } \times \text { recall }}{\text { precision+recall }}$. 

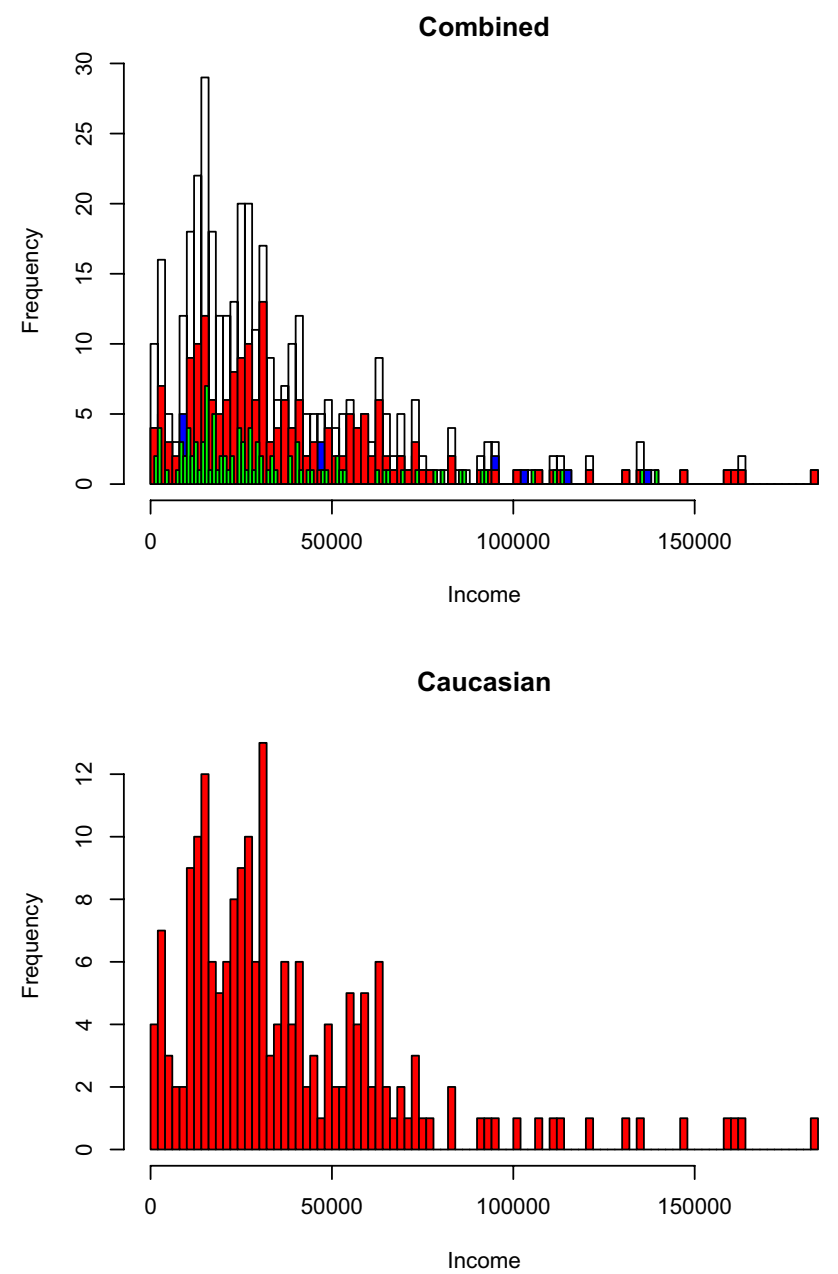

Fig. 3 This figure presents four histograms. These histograms have been obtained using altered data. On the top left-end corner, four histograms are represented simultaneously, showing the distribution of income in the data set along with the distribution of income of each

\subsection{Ethnicity set}

For the "Ethnicity Set", in a first step, we will assess the suitability of the data set for scoring purposes. Therefore, the sample is split in two subsamples; $75 \%$ of the initial set is used for training purposes and $25 \%$ for testing purposes. As the response variable is continuous, to assess the suitability of the data set, we will perform a regression. The mse obtained is equal to 0.008210515 , supporting the conclusion that the data set is adequate for scoring purposes.

In a second step, we will now be using an identical data set to predict the ethnic group of the customers, facing now a classification problem, we obtained a F1-score equal to 0.6507937 . This result demonstrates that the data are already containing a lot of information regarding the ethnic
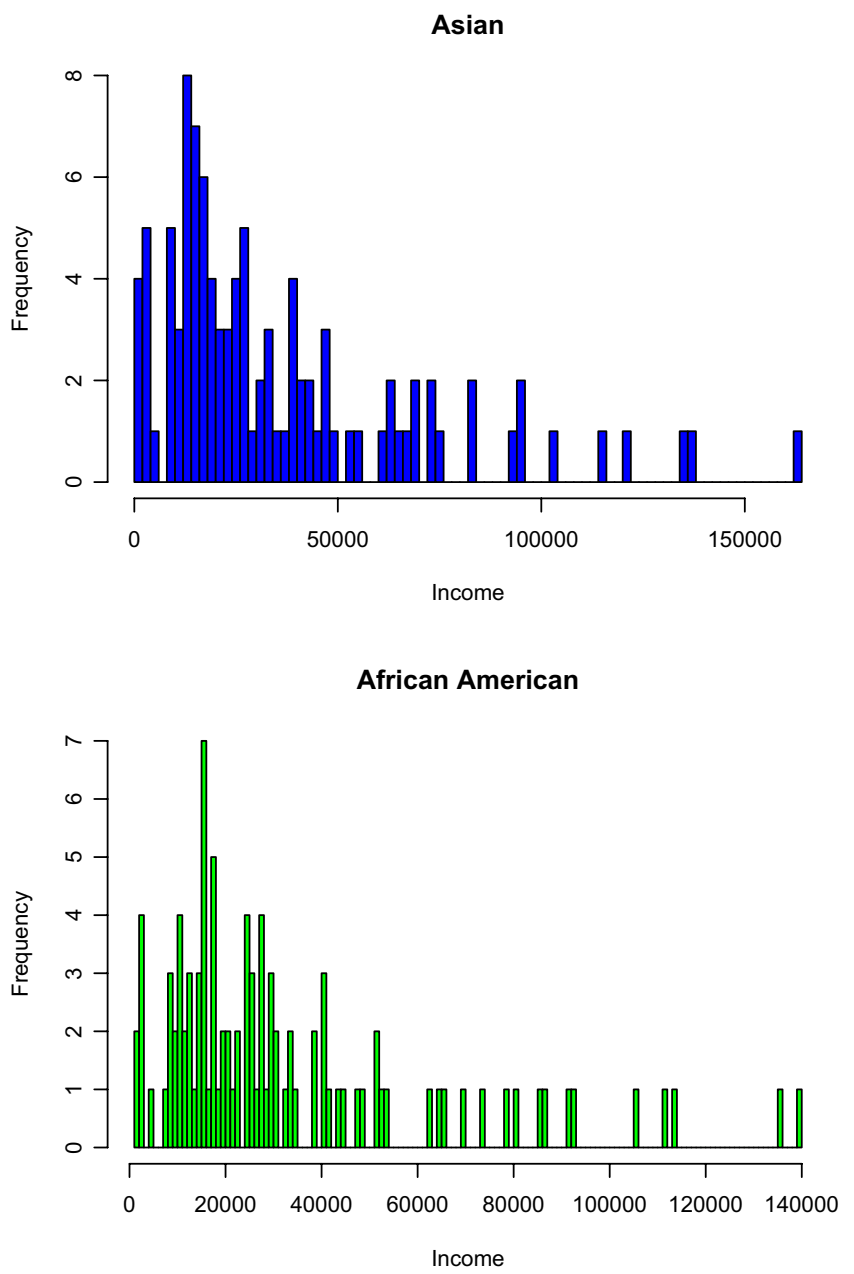

ethnic group. The three other histograms depict the distribution of income for each ethnic group, i.e., Caucasian, African-American, and Asian

affiliation of the bank's customers. Figure 6 also provides the weight of each variable in the predictions, and it appears that the factors related to the financial wealth of the applicants are predominant, i.e., the current credit limit, the money available on their bank account, and their income. Thus, it is not surprising that people earning less money face a lower access to credit.

In a third step, to further test our hypothesis, we will try to predict the ethnic group of each customer contained in the data set after modifying the data to better reflect the reality. After modifying the revenues of the different ethnic groups as well as the related elements such as their ratings, when we tried to reclassify, the results were spectacular, the quality of the classification as given by the F1-score was 0.7 , and went up 0.9863014 when we implemented an oversampling 


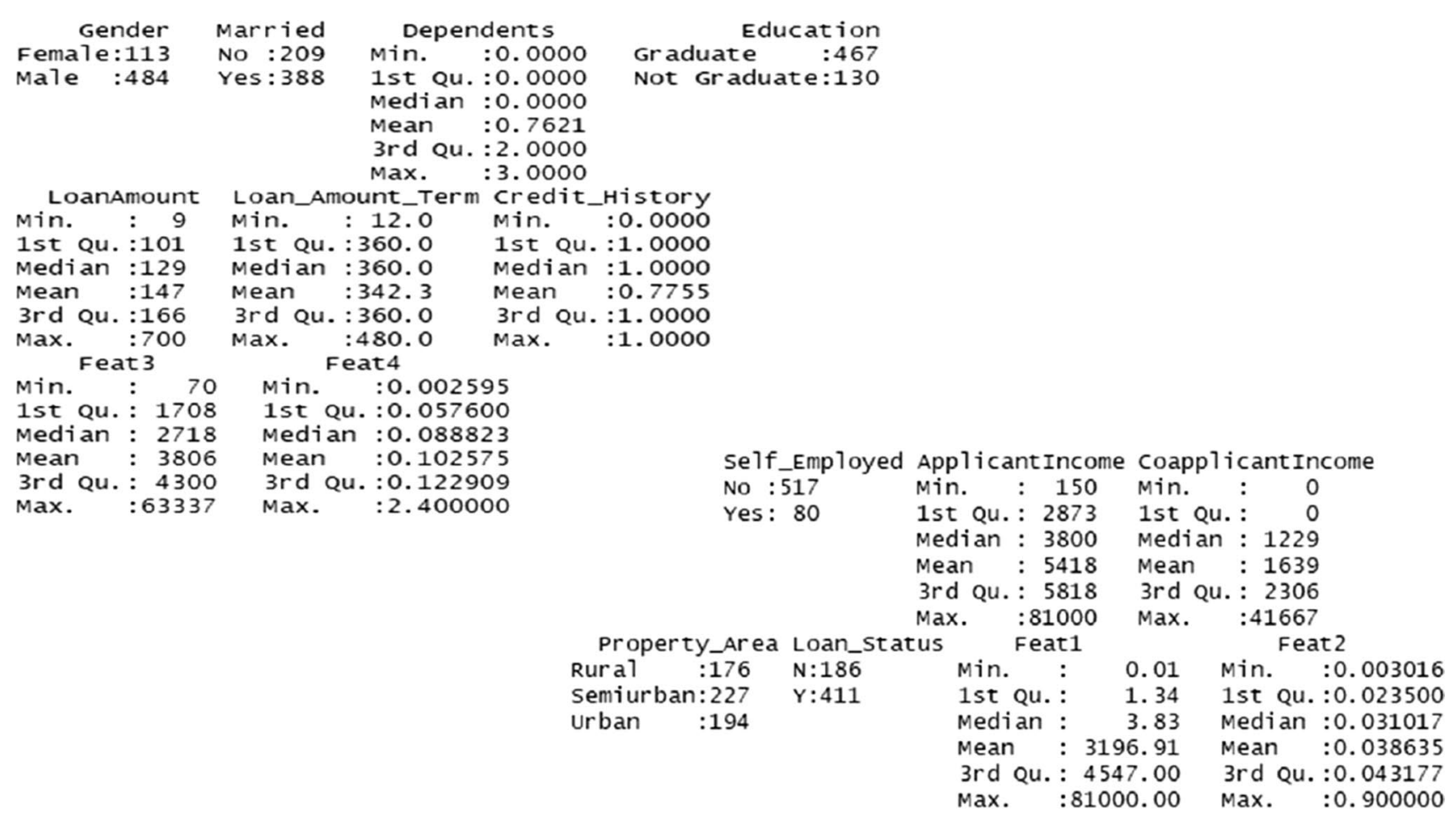

Fig. 4 This table provides the descriptive statistics of the "Gender Set". This data set contains 597 data points; 113 of these represent women and 484 of these represent man. $31 \%$ of the applications contained in the data set have lead to a refusal

strategy to rebalance the data set, i.e., creating synthetic data points in such a way that the three ethnic groups are represented by a population of similar sizes (see Table 4).

As a conclusion, the information transmitted to financial institutions when applying for a loan contains sufficient information to figure out the ethnic group of the customers, and the pertaining biases mechanically transmitted into their evaluation.

\subsection{Gender Set}

As for the "Ethnicity Set", the "Gender Set" was split: $75 \%$ of the initial set was used for training purposes and $25 \%$ for testing purposes. Once again, in the first step, we checked if the data set was adequate for credit scoring purposes. The results regarding the loan approval predictions are provided in Table 4. The initial F1-score obtained is equal to 0.5052632 which is not sufficient to validate the hypothesis. We assumed that feature engineering might improve the algorithm performance, but, once again, the F1-score obtained was equal to 0.5 , which is not sufficient to validate this subsequent hypothesis. After further investigation, we noticed that the data set was unbalanced, i.e., there was a lot more approvals than refusals (however, not unbalanced enough to provide unreliable results) in the data set. To overcome that issue, we implemented an SMOTE strategy allowing rebalancing the data set. The SMOTE approach was implemented to increase the size of the information set related to unapproved loans. Following this procedure, the F1-score increased to 0.8295189 . Adding up feature engineering, the F1-score went up to 0.843418 (see Table 5). Therefore, the data set can be used for scoring purposes. Figure 7 presents the "variable importance" graph, showing that on this data set, the applicant income is one of the main factors driving the results.

Considering the prediction of customers' gender, the results are following the same patterns. The F1-score obtained on the raw data is equal to 0.3333333 . To improve the quality of the adjustments, the following features have been engineered:

1. Applicant income/(co-applicant income +1$)$

2. Loan amount/applicant income

3. Applicant income/(dependents +1$)$

4. Loan amount term/applicant income.

Using feature engineering, the result of the F1-score is equal to 0.3666667. However, with the SMOTE approach, the result increased to 0.8583765 , and to 0.8773748 (see Table 6), once the features engineered had been added. Therefore, the same data set can be used for gender prediction purposes. 
Combined

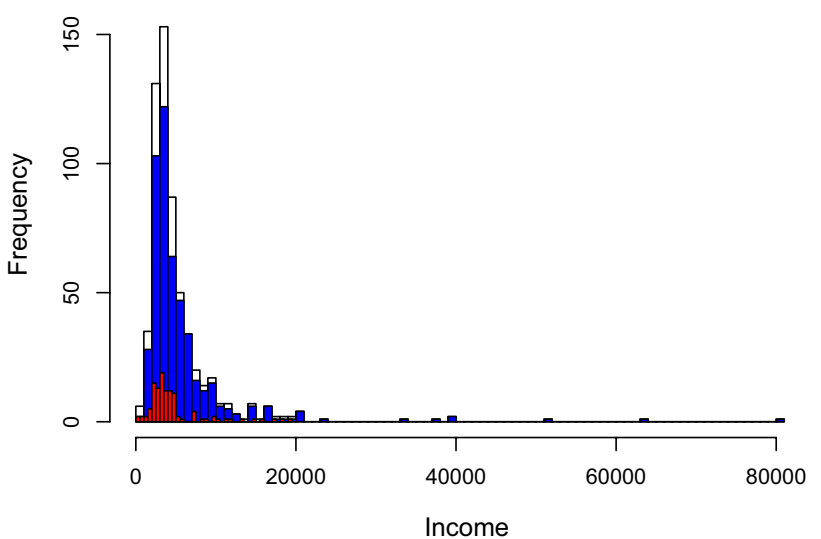

Man

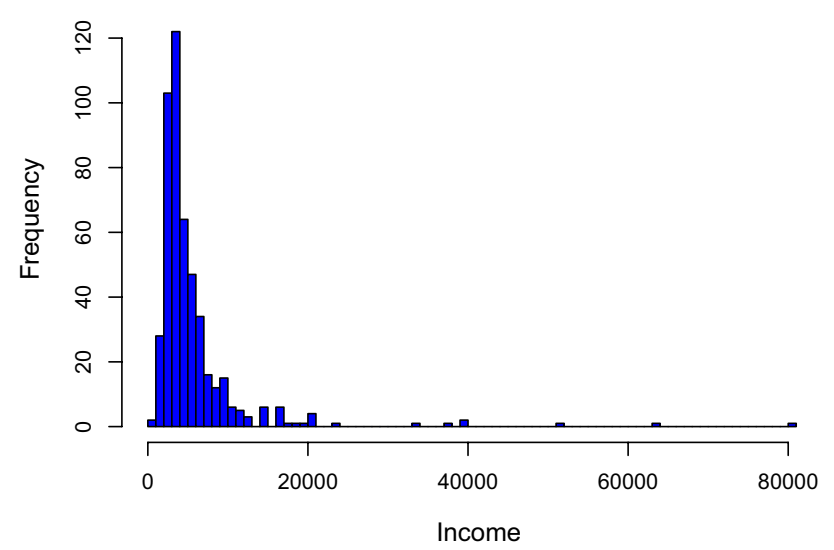

Fig. 5 This figure presents four histograms. On the top left-end corner, the various groups are represented simultaneously. The distribution of income of the whole data set is depicted along the distribution of income of each gender. Two other histograms depict the distribu-

Table 3 Quartiles of both women and men distributions of income

\begin{tabular}{lllll}
\hline $0 \%$ & $25 \%$ & $50 \%$ & $75 \%$ & $100 \%$ \\
\hline $\begin{array}{c}\text { Women } \\
210\end{array}$ & 2870 & 3655 & 4727 & 19,484 \\
$\begin{array}{c}\text { Men } \\
150\end{array}$ & 2980 & 3859 & 5827 & 81,000 \\
\hline
\end{tabular}

\section{Conclusion}

In this paper, our objective was to assess if social biases were captured into credit scoring, and the assumption which we made was that if social biases were not included, then factors characterizing credit scores would be sufficiently different from those that can characterize either men, women,
Combined (Tail)

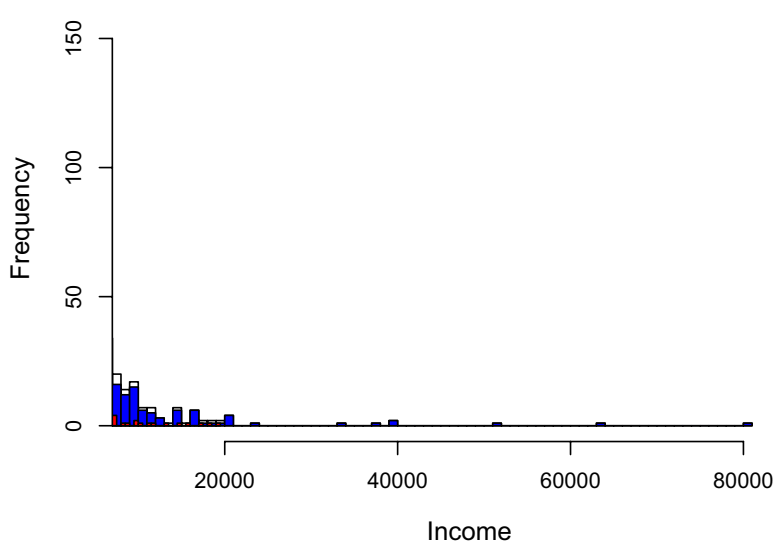

Woman

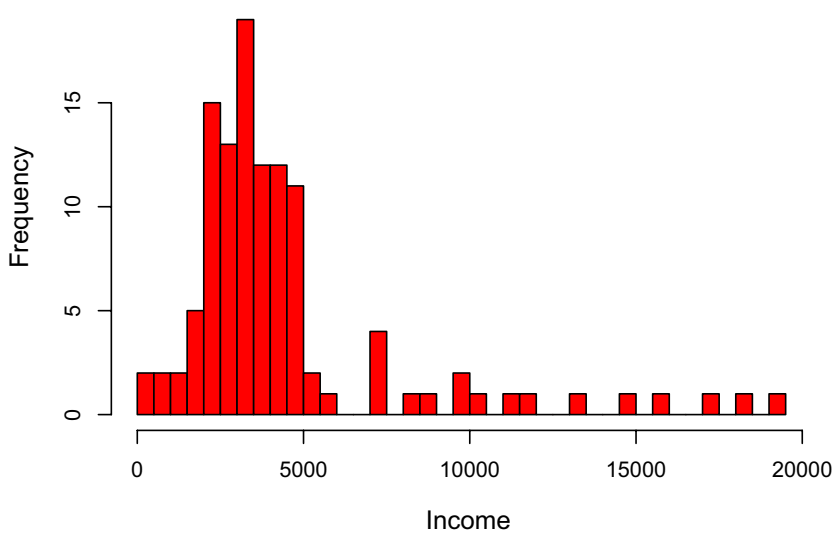

tion of income of each gender group (bottom left and bottom right). The histogram located in the top right-hand corner represents the tail of the income distribution, showing that over a certain threshold, women are not represented anymore

or any ethnic groups. If the data used to score customers can be used to predict any sensitive information, and if the data are socially biased, then the credit score will also be biased.

The most interesting part of the analysis is the fact that results obtained to score customers can be used to predict if the gender or the ethnicity of the customers, and thus, all social biases translated in the data are mechanically included in the scores, and therefore, discrimination is mechanically translated into loan supply, and kept in the data sets for training purposes, ensuring that such discrimination continues and is potentially reinforced. Through that mechanism, social biases become societal biases, as driven by the norm.

What is quite interesting is that it could be possible to unbias the datasets; however, if we consider that a customer with a lower revenue is riskier for a bank than a customer with a higher revenue, then correcting the biases 
Fig. 6 This figure presents the graph of "variable importance" for the "Ethnicity Set". It is interesting to note that the graph confirms the fact that the three most important variables are all related to the financial wealth of customers

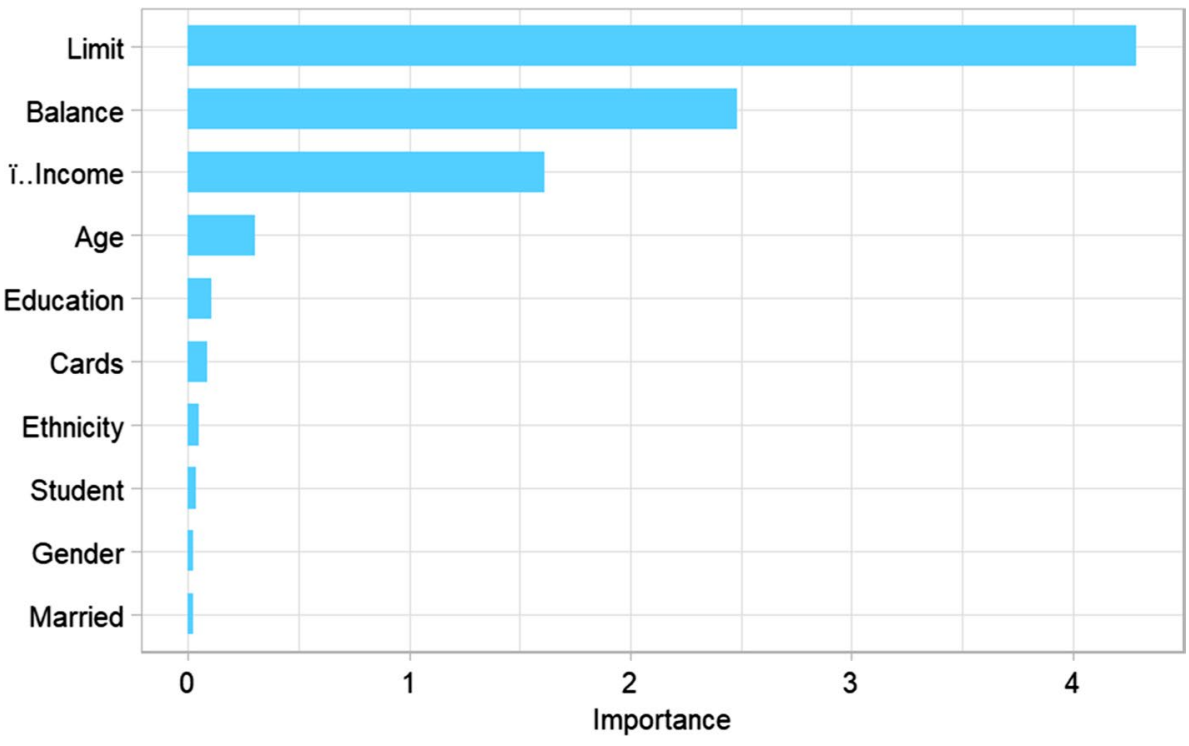

Table 4 F1-score obtained for the random forest classification performed using the "Ethnicity Set" for ethnicity prediction purposes

Data as provided

Data modified

Data modified smote
0.6507937

0.7

0.9863014 by ensuring that social biases are not captured in the data could lead financial institutions to take higher risks. Thus, one may wonder if the solution would not come from the regulator itself. Another aspect appeared in this analysis, if the data set is homogeneous, it becomes complicated to predict either the gender or the ethnic groups, though it would still be possible to score the customer.
Fig. 7 This figure presents the graph of "variable importance" for the "Gender Set". As for the "Ethnicity Set", the most important variables are related to customers financial wealth

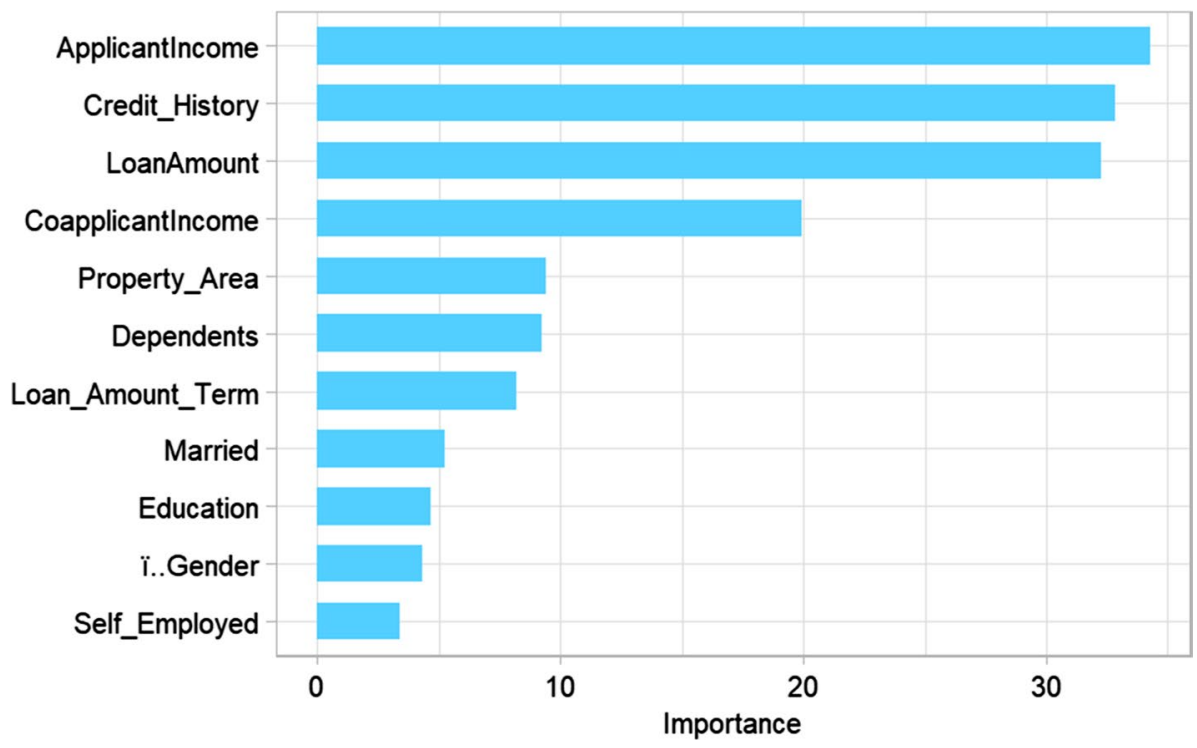


Table 5 F1-Score obtained for the random forest classification performed using the "Gender Set" for loan approval prediction purposes. Once the resampling strategy (SMOTE) has been applied, the performance of the algorithm is sufficient to precisely predict customers' loan approvals

\begin{tabular}{ll}
\hline & Random forest \\
\hline Data as provided & 0.5052632 \\
Features engineered & 0.5 \\
Smote & 0.8295189 \\
Smote features engineered & 0.843418 \\
\hline
\end{tabular}

Table 6 F1-score obtained for the random forest classification performed using the "Gender Set" for gender prediction purposes. Once the resampling strategy (SMOTE) has been applied, the performance of the algorithm is sufficient to precisely predict customers' gender

\begin{tabular}{ll}
\hline & Random forest \\
\hline Data as provided & 0.3333333 \\
Features engineered & 0.3666667 \\
Smote & 0.8583765 \\
Smote features engineered & 0.8773748 \\
\hline
\end{tabular}

Unfortunately, this might lead to fully unbalanced subsamples in which we would have non-approved loans on one side and approved loans on the other. Unbiasing either the data set or the algorithm will be the topic of our next paper, though we will have to address the issue carefully considering that unbiasing a data set is likely to engender an opposite bias.

Open Access This article is licensed under a Creative Commons Attribution 4.0 International License, which permits use, sharing, adaptation, distribution and reproduction in any medium or format, as long as you give appropriate credit to the original author(s) and the source, provide a link to the Creative Commons licence, and indicate if changes were made. The images or other third party material in this article are included in the article's Creative Commons licence, unless indicated otherwise in a credit line to the material. If material is not included in the article's Creative Commons licence and your intended use is not permitted by statutory regulation or exceeds the permitted use, you will need to obtain permission directly from the copyright holder. To view a copy of this licence, visit http://creativecommons .org/licenses/by/4.0/.

\section{References}

1. Addo, P.M., Guegan, D., Hassani, B.: Credit risk analysis using machine and deep learning models. Risks 6(2), 38 (2018)

2. Breiman, L.: Random forests. Mach. Learn. 45(1), 5-32 (2001)

3. Guegan, D., Hassani, B.: Regulatory learning: how to supervise machine learning models? An application to credit scoring. J. Financ. Data Sci. 4(3), 157-171 (2018)

4. Hall, A.V., Hall, E.V., Perry, J.L.: Black and blue: exploring racial bias and law enforcement in the killings of unarmed black male civilians. Am. Psychol. 71(3), 175 (2016)

5. Hegewisch, A., Hartmann, H.: The Gender Wage Gap: 2018; Earnings Differences by Race and Ethnicity, vol. 7. Institute for Women's Policy Research, Washington (2018)

6. Holmes, T.E.: Credit card race, age, gender statistics. creditcards. com (2019). https://www.creditcards.com/credit-card-news/raceage-gender-statistics/

7. Levinson, J.D., Smith, R.J.: Implicit Racial Bias Across the Law. Cambridge University Press, Cambridge (2012)

8. ONS: Ethnicity Pay Gaps in Great Britain: 2018. Office of National Statistics, Cardiff (2019)

9. Witzany, J.: Credit risk management. In: Credit Risk Management, pp. 5-18. Springer, Berlin (2017) 